\title{
Usefulness of Dual Volume Reconstruction of Three- dimensional Rotational Angiography in the Surgical Treatment of Spinal Vascular Malformations: Assessment of Image Similarity Between Angiography and Microscopy
}

\author{
Dae Han CHOI, Chan Jong YOO, Woo Kyung KIM, Sang Gu LEE, Seong SON, Myeong Jin KIM \\ Gachon University College of Medicine, Gil Medical Center, Department of Neurosurgery, Incheon, Republic of Korea
}

Corresponding author: Myeong Jin KIM skymedi@gachon.ac.kr

\section{ABSTRACT}

AIM: To describe and demonstrate the close analogy between dual volume reconstruction images derived using three-dimensional digital subtraction angiography (3D-DSA) and surgical microscopic images.

MATERIAL and METHODS: From 2013 to 2018, 12 patients with spinal vascular malformation (SVM) underwent preoperative 3D-DSA with dual volume reconstruction followed by surgery for the SVM without prior endovascular treatment. Two spinal neurosurgeons involved in each operation were surveyed regarding the similarity between the dual volume images of 3D-DSA and the surgical microscopic images with respect to the following four aspects: (1) relationship between bony structures and the entry artery going to the feeder; (2) feeding artery; (3) shunting point or nidus location; and (4) draining vein.

RESULTS: The patients were diagnosed with spinal dural arteriovenous fistulas $(n=8)$ and spinal arteriovenous malformations $(n=4)$. Two, six, and four lesions showed cervical, thoracic, and lumbar area distribution, respectively. All operations were successful and without complications. The correspondence between dual volume images and intraoperative microscopic images, with respect to bony structure and entry artery, showed perfect reliability $(\kappa=1.000 ; p=0.000)$ and a high level of similarity in all cases. With respect to the feeding artery, shunt point/nidus location, and draining vein, there was substantial reliability between the operator and the observer $(\kappa=0.750 ; p=0.045)$.

CONCLUSION: Images obtained using the dual volume reconstruction technique of the 3D-DSA exhibit a reliable similarity to real microscopic images and are useful in the surgical treatment of SVMs with respect to surgical planning, targeting, and orientation.

KEYWORDS: Angiography, Spine, Arteriovenous fistula, Arteriovenous malformation, Surgery

\section{INTRODUCTION}

O pinal vascular malformations (SVMs), including spinal dural arteriovenous fistula (SDAVF) and spinal arteriovenous malformation (SAVM), are rare and complex; their treatment is challenging $(7,11,17,22)$. Recently, endovascular treatment has become more popular than surgical treatment; however, the endovascular approach is not always possible owing to inaccessible routes and predicted fatality due to the treatment $(1,23)$. SVMs may have tortuous and vague feeder vessels that are the only accessible route; in such cases, the surgical approach may be more intuitive and favorable than the endovascular approach. However, the surgical approach might also be fatal if the surgeon misjudges the pathological vessel because of complexity. Studies have acknowledged
Dae Han CHOI (1) : 0000-0001-5281-2770 Chan Jong YOO (1): :0000-0002-2680-7692 Woo Kyung KIM (1) : 0000-0002-0974-903X

\author{
Sang Gu LEE (1D : 0000-0001-9943-4906 \\ Seong SON (D): 0000-0002-2815-9908 \\ Myeong Jin KIM (1) : 0000-0001-5855-5533
}


three-dimensional digital subtraction angiography (3D-DSA) for the successful results of many treatment modalities $(8,14,22)$, as 3D-DSA provides unrestricted angle views for the surgeon prior to the operation and the key point of the curable target vessel. Intraoperative angiography and intraoperative indocyanine green videoangiography (ICGVA) may also be more useful after coagulation or clipping of the fistulous site than prior to it $(24,26)$. However, intraoperative angiography is a challenge to perform because of the prone position of the patient; thus, ICGVA may be a more attractive option for improving surgical results during the operation $(21,24-26)$. Some investigators have highlighted the usefulness of 3D-DSA for the endovascular approach in the treatment of spinal vascular lesions $(18,19,22)$. However, there have been few reports on the utility of 3D-DSA in the surgical treatment of spinal vascular lesions, especially in terms of the dual volume reconstruction technique. In this technique, two acquired data volumes are reconstructed separately and then fused together. The dual volume reconstruction technique was developed to minimize artifacts and show a greater level of detail in intracranial treatment modalities $(2,6)$. Moreover, the usefulness and efficacy of this technique have rarely been reported for surgical or endovascular treatments of intracranial vascular lesions such as aneurysms or dural arteriovenous fistulas $(2,6,10)$. Hence, in this study, we aimed to describe and demonstrate the parallels between dual volume reconstruction images derived using 3D-DSA and surgical microscopic images.

\section{MATERIAL and METHODS}

\section{Study Population}

From 2013 to 2018, 12 patients were diagnosed with SDAVF or SAVM in a single institute. All of them underwent 3D-DSA with dual volume reconstruction during diagnostic and preoperative conventional catheter angiography. The preferred modality of treatment was open surgery if both the surgical and endovascular approach were possible, and all patients underwent surgery for the SVM without prior endovascular treatment. This study was approved by the local institutional review board (No. GAIRB2019-139).

\section{Angiographic Procedure}

The 3D-DSA images were acquired in rotational angiography using the single A-plane of a biplane angiographic unit (Axiom Artis zee, Siemens Healthcare, Erlangen, Germany). The rotational angle was $200^{\circ}$ and the rotational speed of the C-arm was $40^{\circ}$ per second. The rotation protocol was performed with the patient under temporary apnea to prevent thoracic and abdominal motion artifacts. Data were acquired in a $1024 \times 1024$ matrix using a $48 \mathrm{~cm}$ field-of-view flat panel detector with contrast material injection rates of 1 to $2 \mathrm{ml}$ per second, resulting in a total of 6-12 $\mathrm{ml}$ of contrast in the early arterial phase. The image data were transferred to a workstation (LEONARDO, Siemens Healthcare, Erlangen, Germany), where construction of 3D images was performed. A complete acquisition consisted of two rotational scans: The first scan collected subtraction masks, and the second acquired images during the passage of a contrast medium.
Software reconstructions then enabled the extraction of residual bony structures, deletion of unnecessary vessels, zooming and rotation of the image, and setting of the final reconstruction image on the pertinent surgical view with respect to the surgeon.

\section{Surgical Procedure}

All operations were performed under general anesthesia and after positioning the patient in the prone position. A posteriorapproached laminotomy, laminectomy, or laminoplasty was performed, as appropriate, according to the surgical anatomy of the lesion observed using microscopy. Intraoperative monitoring (IOM) was evaluated throughout the procedures in all cases. The surgical approach was based on the dual volume reconstruction image oriented toward a surgical view. After detection and obliteration of the shunt point, ICGVA was performed and the result of the operation was confirmed immediately.

\section{Inter-Surgeon Reliability Assessment}

Two spinal neurosurgeons involved in each operation were surveyed postoperatively to answer 'Yes' or 'No' regarding the similarity between the 3D-DSA dual volume images and the surgical microscopic images with respect to four aspects: (1) relationship between bony structures and the entry artery going to the feeder; (2) feeding artery; (3) shunt or nidus location; and (4) draining vein. One surgeon, as the operator, answered the survey postoperatively, and another rater, as an observer, completed the survey after viewing a video of a partial scene of the operation recorded before coagulation or clipping of the target vessel.

\section{Statistical Analysis}

For inter-surgeon reliability analysis, Cohen's kappa (к) was used to assess repeat measurement agreement for each operator. Agreement measured by $\kappa$ is interpreted as almost perfect when the $\kappa$ value is between 0.81 and 1.00 , substantial when the $\kappa$ values are between 0.61 and 0.80 , moderate when the $\kappa$ values are between 0.41 and 0.60 , fair when the $\kappa$ values are between 0.21 and 0.40 , and poor when the $\kappa$ values are between 0 and 0.20 (12). $p$ values $<0.05$ were considered to be significant. Statistical analyses were performed using SPSS version 23 (IBM, Armonk, NY, USA).

\section{RESULTS}

The average age of the patients studied was 49.7 years (range 29-65 years). The distribution by sex was balanced, with a male:female ratio of 2.0 (8:4). The patients were diagnosed with SDAVFs $(n=8)$ and SAVMs $(n=4)$. Two, six, and four lesions showed cervical, thoracic, and lumbar area distribution, respectively. A detailed description of the lesions is presented in Table I. All operations were successful without disorientation of the spine level and postoperative complications.

The concordance between responders with respect to bony structure and the entry artery showed perfect reliability $(\kappa=$ $1.000 ; p<0.001$ ) and a high level of similarity in all cases (Table II). With respect to the feeding artery, shunt/nidus location, 
Table I: Basic Characteristics of 12 Patients with Spinal Vascular Malformation Who Underwent Surgical Treatment

\begin{tabular}{ccccccc}
\hline No. & $\begin{array}{c}\text { Sex/Age } \\
\text { (year) }\end{array}$ & Diagnosis & Location & $\begin{array}{c}\text { Foraminal feeder } \\
\text { starting point }\end{array}$ & $\begin{array}{c}\text { Shunt or } \\
\text { nidus level }\end{array}$ & Feeder \\
\hline 1 & M/63 & Spinal dural AVF & Lumbar & T12/L1 & L1 & Right L1 radicular artery \\
\hline 2 & F/33 & Perimedullary spinal AVM & Lumbar & L3/L4 & L3/4 & PSA arising from left L3 segmental artery \\
\hline 3 & M/32 & Perimedullary spinal AVM & Thoracic & T9/T10 & T12 & ASA arising from left T9 intercostal artery \\
\hline 4 & F/50 & Spinal dural AVF & Thoracic & T8/T9 & T4/5 & ASA arising from left T8 intercostal artery \\
\hline 5 & M/52 & Perimedullary spinal AVM & Thoracic & T9/T10 & T8/9 & ASA arising from left T9 intercostal artery \\
\hline 6 & M/55 & Spinal dural AVF & Thoracic & L1/L2 & T12/L1 & ASA arising from right L1 intercostal \\
artery
\end{tabular}

AVF: Arteriovenous fistula, AVM: Arteriovenous malformation, ASA: Anterior spinal artery, PSA: Posterior spinal artery, VA: Vertebral artery. *representative cases.

Table II: Inter-Surgeon Agreement of Similarity Between the Dual Volume Images and the Surgical Microscopic Views Based on 4 Categories

Questionnaire categories and answers of each surgeon

Shunt point or nidus location
Bony structure and an entry artery
Draining vein

\begin{tabular}{ccccccccc}
\hline No. & Operator & Observer & Operator & Observer & Operator & Observer & Operator & Observer \\
\hline 1 & $\mathrm{Y}$ & $\mathrm{Y}$ & $\mathrm{Y}$ & $\mathrm{Y}$ & $\mathrm{Y}$ & $\mathrm{Y}$ & $\mathrm{Y}$ & $\mathrm{Y}$ \\
\hline 2 & $\mathrm{Y}$ & $\mathrm{Y}$ & $\mathrm{Y}$ & $\mathrm{Y}$ & $\mathrm{Y}$ & $\mathrm{Y}$ & $\mathrm{Y}$ & $\mathrm{Y}$ \\
\hline 3 & $\mathrm{Y}$ & $\mathrm{Y}$ & $\mathrm{Y}$ & $\mathrm{N}$ & $\mathrm{Y}$ & $\mathrm{Y}$ & $\mathrm{Y}$ & $\mathrm{N}$ \\
\hline 4 & $\mathrm{Y}$ & $\mathrm{Y}$ & $\mathrm{Y}$ & $\mathrm{Y}$ & $\mathrm{Y}$ & $\mathrm{N}$ & $\mathrm{Y}$ & $\mathrm{Y}$ \\
\hline 5 & $\mathrm{Y}$ & $\mathrm{Y}$ & $\mathrm{Y}$ & $\mathrm{Y}$ & $\mathrm{Y}$ & $\mathrm{Y}$ & $\mathrm{Y}$ & $\mathrm{Y}$ \\
\hline 6 & $\mathrm{Y}$ & $\mathrm{Y}$ & $\mathrm{Y}$ & $\mathrm{Y}$ & $\mathrm{Y}$ & $\mathrm{Y}$ & $\mathrm{Y}$ & $\mathrm{Y}$ \\
\hline 7 & $\mathrm{Y}$ & $\mathrm{Y}$ & $\mathrm{Y}$ & $\mathrm{Y}$ & $\mathrm{Y}$ & $\mathrm{Y}$ & $\mathrm{Y}$ & $\mathrm{Y}$ \\
\hline 8 & $\mathrm{Y}$ & $\mathrm{Y}$ & $\mathrm{N}$ & $\mathrm{N}$ & $\mathrm{N}$ & $\mathrm{N}$ & $\mathrm{N}$ & $\mathrm{N}$ \\
\hline 9 & $\mathrm{Y}$ & $\mathrm{Y}$ & $\mathrm{Y}$ & $\mathrm{Y}$ & $\mathrm{Y}$ & $\mathrm{Y}$ & $\mathrm{Y}$ & $\mathrm{Y}$ \\
\hline 10 & $\mathrm{Y}$ & $\mathrm{Y}$ & $\mathrm{Y}$ & $\mathrm{Y}$ & $\mathrm{Y}$ & $\mathrm{Y}$ & $\mathrm{Y}$ & $\mathrm{Y}$ \\
\hline 11 & $\mathrm{Y}$ & $\mathrm{Y}$ & $\mathrm{N}$ & $\mathrm{N}$ & $\mathrm{N}$ & $\mathrm{N}$ & $\mathrm{N}$ & $\mathrm{N}$ \\
\hline 12 & $\mathrm{Y}$ & $\mathrm{Y}$ & $\mathrm{Y}$ & $\mathrm{Y}$ & $\mathrm{Y}$ & $\mathrm{Y}$ & $\mathrm{Y}$ & $\mathrm{Y}$ \\
\hline Reliability & $\mathrm{K}=1.000 ; p=0.000$ & $\kappa=0.750 ; p=0.045$ & $\kappa=0.750 ; p=0.045$ & $\kappa=0.750 ; p=0.045$ \\
\hline
\end{tabular}

$Y=$ Yes: being able to find correspondence between the dual volume images and the surgical microscopic images

$\boldsymbol{N}=\mathbf{N o}$ : being unable to find correspondence between the dual volume images and the surgical microscopic images

Strength of inter-surgeon agreement: $\kappa=0.81-1.00$ (almost perfect); $\kappa=0.61-0.80$ (substantial); $\kappa=0.41-0.60$ (moderate); $\kappa=0.21-0.40$ (fair); $\kappa=0$ 0.20 (poor). 


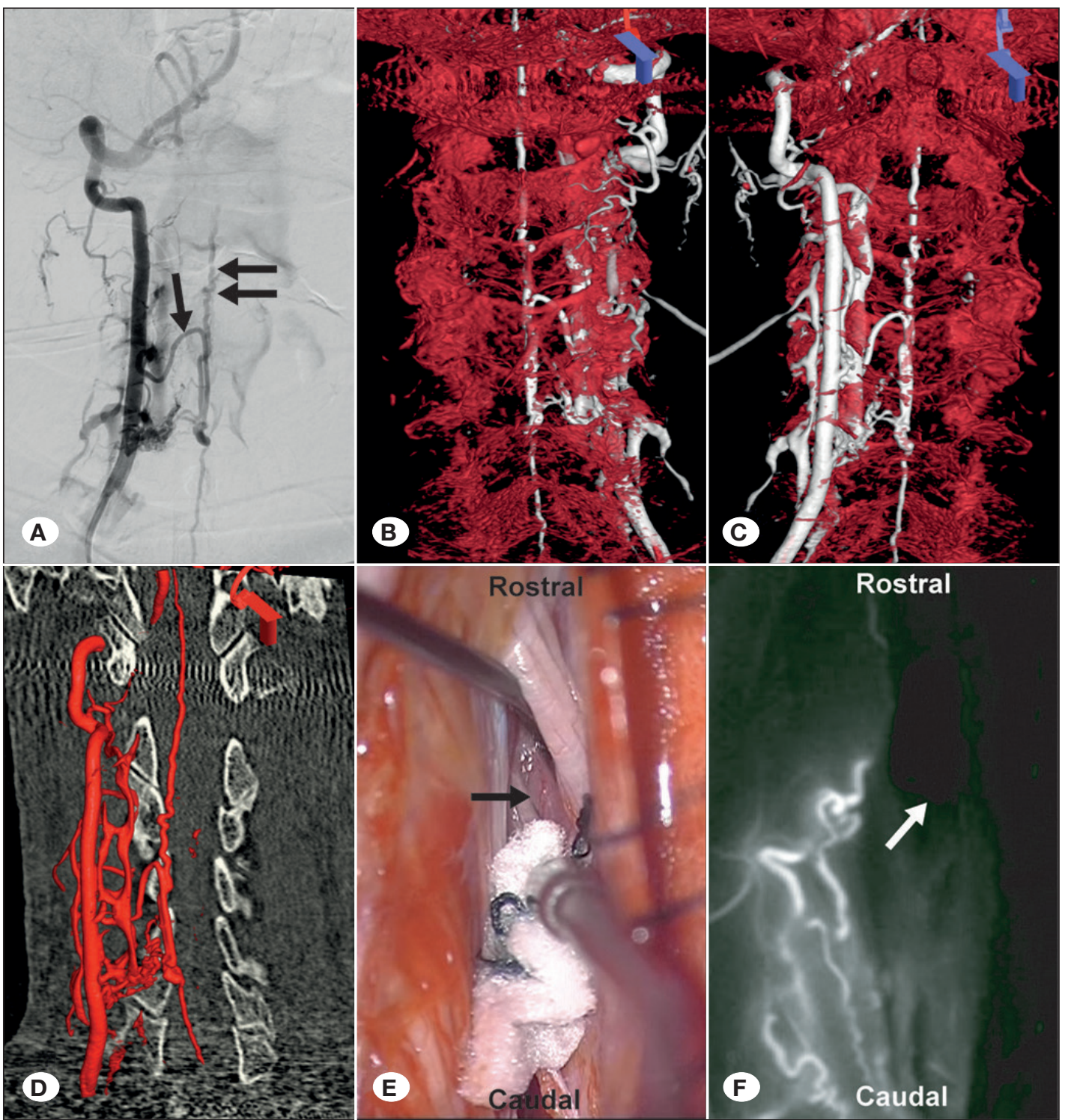

Figure 1: A 65-year-old man presented with cervical spinal dural arteriovenous fistula (patient \# 8). A) Selective digital subtraction angiography (DSA) at the right vertebral artery showed an arteriovenous fistula with shunt from the radiculomedullary artery (arrow) to the draining vein (double arrow) in a cephalad direction at the $\mathrm{C} 6$ level. B-D) Three-dimensional DSA was performed with dual volume reconstruction.

E) The right radiculomedullary artery (arrow) as the single afferent feeder of the fistula was detected at the foramen between $\mathrm{C} 4$ and $\mathrm{C} 5$ after laminectomy and durotomy from $\mathrm{C} 4$ through $\mathrm{C6}$.

F) Indocyanine green videoangiography shows the clipped afferent artery (arrow) without staining. and draining vein, there was substantial reliability between the surgeon and the observer $(\kappa=0.750 ; p=0.045)$. The operator reported high similarity for 10 cases $(83.3 \%)$ and the observer for 9 cases $(75.0 \%)$.

\section{Representative Cases}

\section{Case 1}

A 65-year-old man presented with a tingling sensation and weakness in the right upper extremity for the past 1 year. Initial T2-weighted magnetic resonance image (MRI) of the cervical spine showed a perimedullary signal void at the ventral portion of the C5-6 level. Selective catheter angiography of the right vertebral artery showed an arteriovenous fistula with a shunt from the radiculomedullary artery to the draining vein with cephalad direction at the C6 level (Figure 1A). Subsequently, 3D-DSA was performed with dual volume reconstruction (Figure 1B-D). Based on the key images, laminectomy and durotomy were performed from $\mathrm{C} 4$ to $\mathrm{C} 6$. The right radiculomedullary artery was determined to be the single afferent feeder of the fistula and was identified in the foramen between $\mathrm{C} 4$ and C5 (Figure 1E). Electromyography (EMG) results and nerve conduction velocity (NCV) were stable during the temporary clipping of the feeder for 15 minutes; then, the feeding artery was permanently coagulated and cut. The post-ICGVA was performed once again to confirm the result (Figure 1F). The patient's symptoms improved, and he managed to return to his job 6 months after the operation.

\section{Case 2}

A 29-year-old woman experienced continuous paresthesia and intermittent weakness of the left leg for 2 years. Initial T2weighted MRI of the spine showed perimedullary flow voids between the lower thoracic and entire lumbar levels. Selective spinal catheter angiography of the left T11 intercostal artery showed an arteriovenous malformation fed from the posterior 

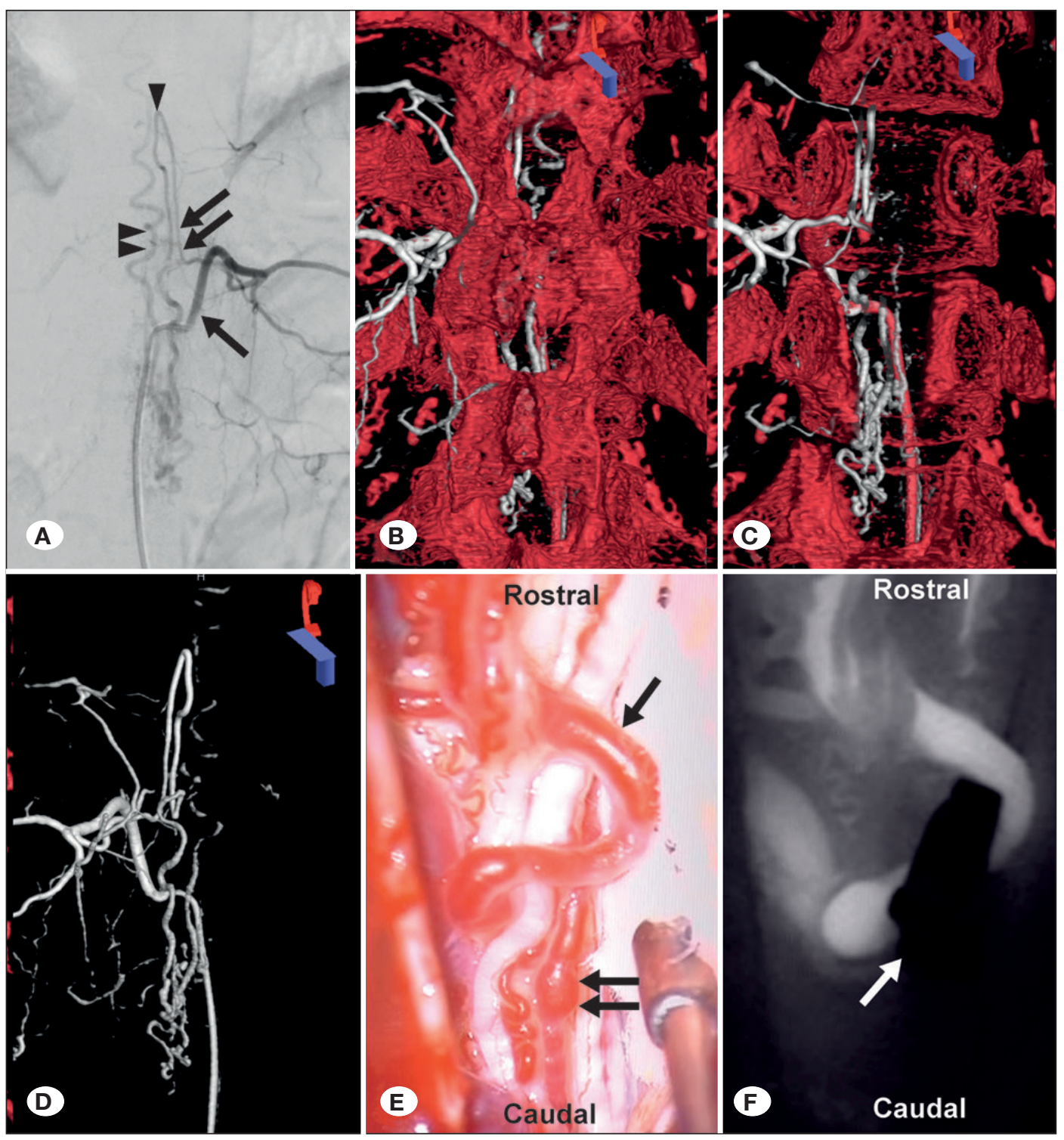

Figure 2. A 29-yearold woman with spinal arteriovenous malformation (patient \# 10).

A) Selective spinal digital subtraction angiography (DSA) at the left T11

intercostal artery (arrow) showed an arteriovenous malformation fed from the posterior spinal artery (arrow head) starting at the left posterior radiculomedullary artery (double arrows) and draining into the perimedullary vein (double arrow heads), with the nidus (white arrow head) located at the L1 level. B-C) Threedimensional DSA was performed with dual volume reconstruction (bone and vasculature) in a surgical view with/without laminas and spinous processes in the pathway of the lesion. D) A single volume reconstructed vessel image was produced.

E) The afferent feeding artery (arrow) and efferent draining vein (double arrows) were identified at the T12-L1 level after laminectomy and durotomy. F) Indocyanine green videoangiography shows the clipped feeder (arrow) without further staining and the draining vein.

spinal artery starting at the left posterior radiculomedullary artery and draining into the perimedullary vein with a nidus located at the L1 level (Figure 2A). Subsequently, 3D-DSA was performed with dual volume reconstruction (bone and vasculature) in a surgical view with/without laminas and spinous processes in the pathway of the lesion (Figure $2 \mathrm{~B}-\mathrm{C}$ ), and a single-volume reconstructed vessel image was also produced (Figure 2D). Based on the surgical view image, laminectomy and durotomy from T11 through L1 level were performed, and the afferent feeding artery was identified (Figure 2E) and coagulated. The target vessel was confirmed using ICGVA after temporary clipping of the feeding artery. The post-ICGVA was performed once again to confirm the result (Figure 2F). During the operation, EMG results and NCV were stable and unchanged. The patient's symptom gradually improved without any complications.

\section{DISCUSSION}

This study demonstrated that preoperative dual volume reconstruction images of 3D-DSA were reliable and corresponded strongly to the microscopic view images during the surgical approach with respect to bony structures, orientation, and location of the target vessels.

Surgical treatment of the SVMs may be more effective in terms of lower recurrence rate than the endovascular treatment if it is possible to completely compromise the shunt of the fistula or perform en bloc resection of the nidus $(3,5)$. To achieve these goals, preoperative planning of the SVM surgery is important to prevent misjudgment and disorientation with regard to the lesion. Especially, a preoperative decision regarding the extent to which surgeons should perform the operation, including the skin incision, laminectomy, and exploration, might be crucial in decreasing intraoperative time and detecting the target lesion. The major challenges of the surgical procedure have been 
localization of the origin of the feeding artery and the draining vein and the risk of error in determining the correct vertebral level (3). Some investigators have reported that the SDAVF level can be preoperatively identified by placing a coil in the feeding artery during preoperative spinal angiography; this technique appears to decrease the risk of error of determining the correct vertebral level (13). However, although there were no complications reported in the literature, the method may not be applicable for all SVMs because of decreased navigability owing to tortuous vasculature and increased risk of thrombosis due to the foreign material. Prestigiacomo et al. reported on the usefulness of 3D-DSA for imaging in SVM (18), and that this kind of information is extremely valuable for spinal surgical procedures and can help determine the most suitable surgical approach (9). Moreover, the surgical view could be always reproduced to represent any angle of the surgeon's view, like in an intracranial lesion (20). In this study, compared to $2 \mathrm{D}$ angiography, dual volume 3D-DSA could additionally and more usefully enable the surgeon to inspect the vascular structure in 360 degree view pre- or intra-operatively in each case. Consequently, the veiled structures (e.g., overlapping vasculature on 2D angiographic image) were identified and distinguished in all cases. The present study showed that the surgical treatments in all cases using the reconstructed surgical view image of the 3D-DSA were successful without incorrect vertebral leveling and complications under presurgical planning. Therefore, we suggest that preoperative planning using the 3D-DSA reconstruction images may be useful and allow treatment without associated risk or difficulty.

The SVMs, surrounded by bony structures, are typically present in a relatively small space with complex angioarchitecture. Moreover, the image quality of the conventional DSA seems to be degraded by many artifacts caused by the technique and physiologic factor including non-homogenous contrast distribution, vessel wall irregularities, pulsation, and motion (8). However, this weakness clearly represents an aspect of the 3D-DSA that can be improved with the single or dual volume reconstruction technique. Single volume reconstruction, composed of two rotational scans that are then fused as one volume, shows simultaneous images including the complex vasculature and bony structure that are difficult to distinguish. The fact that it is hard to separate the two volumes one by one may have some drawbacks including the invisibility of the target vessel overlapped by bony structures, and the obscuring of the shunt point and the nidus adjacent to the bony structure. This study showed that the dual volume images were significantly reliable in terms of similarity with the practical angioarchitecture. Comparatively, in dual volume reconstruction, the acquired data volumes (mask run and contrast run) are reconstructed separately and then fused together, with each volume (e.g., a separate volume for the vessels and another for the devices) being individually modifiable to give the desired contrast, windowing, and color coding (2). Therefore, the dual volume reconstruction technique may help the surgeon better understand the entire architecture composed of the vasculature and the bony structures, and this may provide reliable localization of the SVM and allow planning for the operation. In particular, when oblique and lateral views were difficult to obtain, 3D-DSA was able to help surgeons determine the anteroposterior relationships between the various vascular structures (18).

Recently, some investigators reported that an intraoperative DSA technique, as an alternative method for identification of SVMs, may be a useful tool to identify shunt points, any remaining lesions, and normal vessel structures $(16,24)$. In particular, ICGVA has emerged as a useful real-time monitoring method $(15,21,25,26)$. However, in case of intraoperative DSA, intraoperative vascular access in spine surgery is challenging because patients are often in the prone position (24). Moreover, intraoperative DSA is associated with complications, including thrombosis, and may interrupt the surgical procedure, as angiography requires equipment such as the C-arm $(4,17)$. Additionally, ICGVA is limited by the fact that only blood vessels on the surface of the spinal cord that are fully exposed to the operative field can be visualized (26). Therefore, with intraoperative angiography, 3D-DSA with the dual volume reconstruction technique may be more helpful than intraoperative angiography alone in completely obliterating the fistula or removing the nidus.

In this study, there were no comparable features between the 3D-DSA images and the microscopic surgical images in two cervical lesion cases (case No. 8 and 11) with respect to the feeding artery, shunting point, and draining vein. Finding the lesion in the ventral cervical cord may be more difficult than in dorsal portions. Moreover, the cervical cord could be less flexible than other segments of the spinal cord; hence, the deep ventral portion may be inaccessible. However, we pre-operatively confirmed the presence of collateral supply vessels to the anterior spinal artery and the afferent pathways in all cases where the SDAVF was identified as a single vessel. In addition, temporary clipping of the target vessel under IOM was useful for confirming the target vessel.

Regarding the difference of opinion between two surgeons, there were two cases (case No. 3 and 4) in which the distance from the foraminal feeder starting level to the shunt/nidus level was relatively greater; in such cases, the angioarchitecture of the complex vasculatures could be compromised and vague. Additional manipulations may be needed on rendering that sharpens the vague outline of the target lesions including vasculature and surrounding structures. Consequently, the dual volume image of the lesion may have less similarity with the surgical microscopic image than with others from the observer's perspective.

\section{Limitations}

The present study was conducted with a small population and there was no comparison of the reliability between single volume reconstruction and dual volume reconstruction. Prior to this study, we anticipated that there would only be a few cases of SVM that would challenge our ability to compare the two groups with abundant cases. Therefore, only the dual volume reconstruction technique was performed in all cases for strengthening reliability.

Another limitation was that the inter-surgeon reliability analysis was performed in uneven situations for each surgeon, 
wherein one was a real-time operator and the other observed a recorded video scene after the operation. However, the observer, not involved in the operation, was only allowed to see a partial scene just before coagulation or clipping of the target vessel. Additionally, the two rating surgeons did not participate in the same operation because of the possibility of interaction between the two surgeons. The other limitation was that the analysis of inter-surgeon agreement may lack reliability because of subjective opinions especially in a small sample. Therefore, a multi-center study with a large case volume and many surgeons should be performed for greater inter-rater reliability.

\section{CONCLUSION}

Images obtained using the dual volume reconstruction technique of 3D-DSA exhibit a reliable similarity to real microscopic images and are useful for the surgical treatment of SVMs with respect to surgical planning, targeting, and orientation. For better reliability of the dual volume reconstruction technique, further studies investigating and comparing it with other pre- or intraoperative imaging methods are required.

\section{REFERENCES}

1. Acerbi F, Ferroli P: Surgery versus embolization in spinal dural arteriovenous fistulas: The ideal competition to improve the care of patients. World Neurosurg 80:E191-E193, 2013

2. Adeeb N, Griessenauer CJ, Patel AS, Moore J, Dolati-Ardejani P, Gupta R, Motiei-Langroudi R, Ogilvy CS, Thomas AJ: Reliability of dual-vs single-volume reconstruction of threedimensional digital subtraction angiography for follow-up evaluation of endovascularly treated intracranial aneurysms. Interv Neuroradiol 22:687-692, 2016

3. Bretonnier M, Henaux PL, Gaberel T, Roualdes V, Kerdiles G, Le Reste PJ, Morandi X: Spinal dural arteriovenous fistulas: clinical outcome after surgery versus embolization: A retrospective study. World Neurosurg 127:e943-e949, 2019

4. Chung JC, Jung SS, Park KS, Ha HG: Intraoperative vertebral artery angiography to guide c1-2 transarticular screw fixation in a patient with athetoid cerebral palsy. J Korean Neurosurg Soc 51:177, 2012

5. Chung SK, Jeon SR, Chung CK, Kim HJ: Surgical treatment of arteriovenous malformations of the spinal cord. J Korean Neurosurg Soc 26:1592-1598, 1997

6. Huang CT, Hsu SK, Su IC: Accurate localization of aneurysm neck margins in clipping of a carotid cave aneurysm using intraoperative dual-volume 3-dimensional volume-rendering rotational angiography. World Neurosurg 101:812. e811-812, e814, 2017

7. Jellema K, Tijssen CC, Sluzewski M, Asbeck FWA, Koudstaal PJ, Gijn J: Spinal dural arteriovenous fistulas-An underdiagnosed disease. J Neurol 253:159-162, 2006

8. Jiang L, Huang CG, Liu P, Yan B, Chen JX, Chen HR, Bai RL, Lu YC: 3-Dimensional rotational angiography for the treatment of spinal cord vascular malformations. Surg Neurol 69:369373; discussion 373-374, 2008
9. Kern M, Naeini R, Lehmann TN, Benndorf G: Imaging of a thoracic spinal nerve haemangioblastoma by threedimensional digital angiography. J Clin Neurosci 13:929-932, 2006

10. Kim MJ, Shin YS, Ihn YK, Kim BM, Yoon PH, Oh SY, Kim BS: Transvenous embolization of cavernous and paracavernous dural arteriovenous fistula through the facial vein: Report of 12 cases. Neurointervention 8(1):15-22, 2013

11. Krings T, Geibprasert S: Spinal dural arteriovenous fistulas. Am J Neuroradiol 30:639-648, 2009

12. Landis JR, Koch GG: The measurement of observer agreement for categorical data. Biometrics 33(1):159-174, 1977

13. Marquardt G, Berkefeld J, Seifert V, Gerlach R: Preoperative coil marking to facilitate intraoperative localization of spinal dural arteriovenous fistulas. Eur Spine J 18:1117-1120, 2009

14. Matsubara N, Miyachi S, Izumi T, Ohshima T, Tsurumi A, Hososhima O, Kinkori T, Yoshida J: Usefulness of threedimensional digital subtraction angiography in endovascular treatment of a spinal dural arteriovenous fistula. J Neurosurg Spine 8:462-467, 2008

15. Misra BK, Samantray SK, Churi ON: Application of fluorescein sodium videoangiography in surgery for spinal arteriovenous malformation. J Clin Neurosci 38:59-62, 2017

16. Nossek E, Chalif DJ, Buciuc R, Gandras EJ, Anderer EG, Insigna S, Dehdashti AR, Setton A: Intraoperative angiography for arteriovenous malformation resection in the prone and lateral positions, using upper extremity arterial access. Oper Neurosurg 13:352-360, 2017

17. Osanai T, Hida K, Asano T, Seki T, Sasamori T, Houkin $\mathrm{K}$ : Ten-year retrospective study on the management of spinal arteriovenous lesions: Efficacy of a combination of intraoperative digital subtraction angiography and intraarterial dye injection. World Neurosurg 104:841-847, 2017

18. Prestigiacomo CJ, Niimi $Y$, Setton A, Berenstein A: Threedimensional rotational spinal angiography in the evaluation and treatment of vascular malformations. Am J Neuroradiol 24:1429-1435, 2003

19. Sciubba DM, Mavinkurve GG, Gailloud P, Garonzik IM, Recinos PF, McGirt MJ, Woodworth GF, Witham T, Khavkin Y, Gokaslan ZL: Preoperative imaging of cervical spine hemangioblastomas using three-dimensional fusion digital subtraction angiography: Report of two cases. J Neurosurg Spine 5:96-100, 2006

20. Siablis D, Kagadis GC, Karamessini MT, Konstantinou D, Karnabatidis D, Petsas T, Nikiforidis GC: Intracranial aneurysms: Reproduction of the surgical view using 3D-CT angiography. Eur J Radiol 55:92-95, 2005

21. Spiotta AM, Bain M, Moskowitz S: Intraoperative indocyanine green angiography as a substitute for conventional angiography in the surgical management of spinal dural arteriovenous fistulae. J Neurointerv Surg 3:182-185, 2011

22. Suh DC, Kim HS, Baek HJ, Park JW, Kim KK, Rhim SC: Angioarchitecture of spinal dural arteriovenous fistula evaluation with 3D rotational angiography. Neurointervention 7:10-16, 2012

23. Sung KS, Song YJ, Kim KU: Novalis stereotactic radiosurgery for spinal dural arteriovenous fistula. J Korean Neurosurg Soc 59:420, 2016 
24. Villelli NW, Lewis DM, Leipzig TJ, DeNardo AJ, Payner TD, Kulwin CG: Intraoperative angiography via the popliteal artery: A useful technique for patients in the prone position. $J$ Neurosurg-Spine 29:322-326, 2018

25. Walsh DC, Zebian B, Tolias CM, Gullan RW: Intraoperative indocyanine green video-angiography as an aid to the microsurgical treatment of spinal vascular malformations. $\mathrm{Br}$ J Neurosurg 28:259-266, 2014
26. Wang GH, Ma GS, Ma J, Hao SY, Li DZ, Han LJ, Wang T, Su W, Han B, Yen CP, Kwan AL: Surgical treatment of spinal vascular malformations performed using intraoperative indocyanine green videoangiography. J Clin Neurosci 20:831-836, 2013 